\title{
Effect of non-equilibrium term in two-particle correlation function on electron-phonon collision integrals
}

\author{
A.Ya. Shul'man \\ Institute of Radio Engineering and Electronics of the RAS, 125009 Moscow, Russia. \\ E-mail:ash@cplire.ru
}

\begin{abstract}
The derivation of kinetic equation for one-particle distribution function $F(p, r, t)$ from BBGKY chain leads to the collision integral expressed in terms of the two-particle correlation function. The latter is in turn expressed by $F$ using Boltzmann's Stosszahlansatz that implies the neglect of initial one-time correlation function $g 2(p 1, p 2, r, t)$ which is non-diagonal in the momentum space. In non-equilibrium case, however, it has been established that pair collisions generate the non-diagonal two-particle correlations that give the essential contribution to the current fluctuations of hot electrons. These correlations have been shown giving also a contribution to the collision integrals, i.e., to kinetic properties of nonequilibrium gas The expression for electron energy loss rate $P$ via phonons is re-derived in detail from this point of view. The order of value of the new term in $P$ in the cases of acoustic- and optical-phonon scattering is evaluated in the electron temperature approximation using the experimental study of hot-electron energy relaxation time in $n-I n S b$ at helium temperatures.
\end{abstract}

Keywords: Kinetic theory, correlation contributions, hot electrons and phonons

PACS: $05.20 . \mathrm{Dd}$, 63.20.Kr, 72.10.Bg, 72.20.Dp, 72.20.Ht

\section{INTRODUCTION}

It was established that pair collisions generate in non-equilibrium case non-zero twoparticle correlations that are non-diagonal in momentum space and give the essential contribution to the current fluctuations of hot electrons [1, 2]. These correlations have been shown giving also a contribution to the collision integrals, i.e., to kinetic properties of non-equilibrium gas [3, 4]. The expression for electron energy loss rate $P$ via phonons is re-derived from this point of view. The formula for $P$ in terms of electron density-density correlation function $\left\langle\rho_{k} \rho_{k}^{+}\right\rangle_{\omega}$ was obtained by Kogan [5] using Fermi Golden Rule. However, in order to come to the well-known result for $P$ the fluctuationdissipation theorem was implicated to express $\left\langle\rho_{k} \rho_{k}^{+}\right\rangle_{\omega}$ in terms of the dielectric function of the electron gas. On this way the non-equilibrium correction has been lost. Here the contribution of the non-diagonal part of the non-equilibrium pair correlator to phonon-electron collision integral and to $P$ is obtained and explicitly calculated in the electron temperature approximation. The contribution obtained does not contain the extra powers of the small parameter of kinetic theory unlike the virial expansions in equilibrium thermodynamics. The order of value of the new term in $P$ is evaluated using the experimental study of hot-electron energy relaxation time in $n-I n S b$ at helium temperatures [6]. 


\section{BASIC DESIGNATION}

Hamiltonian

$H=H_{e}+H_{p}+V_{e p}$

Electron part

$H_{e}=\sum_{\mathbf{k}} \hbar \Omega_{\mathbf{k}} a_{\mathbf{k}}^{+} a_{\mathbf{k}}+V_{e e}$,

where $V_{e e}$ stands for the Coulomb electron-electron interaction

Phonon part

$H_{p}=\sum_{\mathbf{f}} \hbar \omega_{\mathbf{f}} b_{\mathbf{f}}^{+} b_{\mathbf{f}}$

and electron-phonon interaction

$V_{e p}=\frac{1}{\sqrt{V_{0}}} \sum_{\mathbf{f}}\left(c_{f} \rho_{\mathbf{f}}^{+} b_{\mathbf{f}}+c_{f}^{*} \rho_{\mathbf{f}} b_{\mathbf{f}}^{+}\right)$,

where $V_{0}$ is the normalizing volume.

Fourier components of the electron density operator

$\rho_{\mathbf{f}}=\sum_{k} a_{\mathbf{k}+\frac{\mathbf{f}}{2}}^{+} a_{\mathbf{k}-\frac{\mathbf{f}}{2}}, \quad \rho_{\mathbf{f}}^{+}=\rho_{-\mathbf{f}}$

Phonon distribution function

$N_{\mathbf{f}}(t)=\left\langle\left(b_{\mathbf{f}}^{+} b_{\mathbf{f}}\right)_{t}\right\rangle$

Electron distribution function

$F_{\mathbf{k}}(t)=\left\langle\left(a_{\mathbf{k}}^{+} a_{\mathbf{k}}\right)_{t}\right\rangle$

The angle brackets denote the averaging with the density matrix corresponding to the noninteracting electrons and phonons at initial instant of time. The subscript $t$ indicates the Heisenberg representation for electron and phonon operators.

\section{KINETIC EQUATION FOR PHONONS}

We obtain the kinetic equation for slow variables $N_{f}(t)$, smoothing Heisenberg equations of motion over mesoscopic time interval. With accuracy to the second-order terms in $e-p$ interaction we have

$$
\frac{\Delta N_{\mathbf{f}}(t)}{\Delta t}=-\frac{1}{\hbar^{2} \Delta t}\left\langle S^{+}(t) \int_{t}^{t+\Delta t} d t \prime \int_{t}^{t \prime} d t \prime \prime\left[V_{e p}^{0}(t \prime \prime),\left[V_{e p}^{0}(t \prime),\left(b_{\mathbf{f}}^{+} b_{\mathbf{f}}\right)_{t}\right]\right] S(t)\right\rangle .
$$

The superscript 0 denotes operators in the interaction representation with respect to the electron-phonon interaction $V_{e p}$.

Calculations of the internal commutator lead to the desired result involving the electron density-density correlation function:

$$
\frac{d N_{\mathbf{f}}}{d t}=\frac{\left|c_{f}\right|^{2}}{V_{0} \hbar^{2}}\left\{\left\langle\rho_{\mathbf{f}}^{+} \rho_{\mathbf{f}}\right\rangle_{-\omega_{f}}^{0}\left(N_{\mathbf{f}}+1\right)-\left\langle\rho_{\mathbf{f}} \rho_{\mathbf{f}}^{+}\right\rangle_{\omega_{f}}^{0} N_{\mathbf{f}}\right\},
$$

where the condition $\omega_{\mathrm{f}} \Delta t>>1$ was used. The function

$$
\left\langle\rho_{\mathbf{f}} \rho_{\mathbf{f}}^{+}\right\rangle_{\omega}=\int_{-\infty}^{\infty} d \tau\left\langle\rho_{\mathbf{f}}(t+\tau) \rho_{\mathbf{f}}^{+}(t)\right\rangle \exp (i \omega \tau)
$$


is the spectral density of the correlation function of the electron density fluctuations.

\section{Spectral density of the high-frequency electron fluctuations in the electron temperature approximations}

The spectral density must be calculated without $V_{e p}$ taken into account. From equations of motion for the electron operators we obtain in the random phase approximation:

$$
\left\langle\rho_{\mathbf{f}}^{+} \rho_{\mathbf{f}}\right\rangle_{\omega}^{0}=\frac{\left\langle\rho_{\mathbf{f}}^{+} \rho_{\mathbf{f}}\right\rangle_{\omega}^{00}}{|\varepsilon(\omega, \mathbf{f})|^{2}},
$$

where $\varepsilon(\omega, \mathbf{f})$ is the relative dielectric function of the electron gas embedded into the dielectric medium and $\left\langle\rho_{\mathbf{f}}^{+} \rho_{\mathbf{f}}\right\rangle_{\omega}^{00}$ is the spectral density of the density-density correlation function for non-interacting electrons. The latter is connected with one-time correlation function $\left\langle\rho_{\mathbf{f}}(\mathbf{k}) \rho_{\mathbf{f}}^{+}\left(\mathbf{k}_{1}\right)\right\rangle_{t}$ by equations of motion for free electrons. The one-time correlation function is defined as

$$
\left\langle\rho_{\mathbf{f}}(\mathbf{k}) \rho_{\mathbf{f}}^{+}\left(\mathbf{k}_{1}\right)\right\rangle_{t} \equiv\left\langle a_{\mathbf{k}-\frac{\mathrm{f}}{2}}^{+} a_{\mathbf{k}+\frac{\mathrm{f}}{2}} a_{\mathbf{k}_{1}+\frac{\mathrm{f}}{2}}^{+} a_{\mathbf{k}_{1}-\frac{\mathbf{f}}{2}}\right\rangle_{t} .
$$

The one-time correlation function is independent of time (in steady state) under following condition on the electron energies $\Omega_{k}=\hbar k^{2} / 2 m$ :

$$
\Omega_{\mathbf{k}-\frac{\mathrm{f}}{2}}-\Omega_{\mathbf{k}+\frac{\mathrm{f}}{2}}+\Omega_{\mathbf{k}_{1}+\frac{\mathrm{f}}{2}}-\Omega_{\mathbf{k}_{1}-\frac{\mathrm{f}}{2}} \equiv \frac{\hbar}{m} \mathbf{f}\left(\mathbf{k}_{1}-\mathbf{k}\right)=0 .
$$

Because $\mathbf{f} \neq 0$ then it is necessary to be $\mathbf{k}_{1}=\mathbf{k}$. Therefore, this condition provides the one-time correlator to be the integral of motion during the time interval $\Delta t$ like the distribution functions $N_{\mathbf{f}}$ and $F_{\mathbf{k}}$.

\section{Kinetic equation with account for the contribution of the non-equilibrium pair correlations}

The substitution of the explicit expression Eq.(2)for correlators to the kinetic equation Eq.(11) gives rise to

$$
\begin{aligned}
\frac{d N_{\mathbf{f}}}{d t} & =\frac{2\left|C_{f}\right|^{2}}{V_{0} \hbar^{2}\left|\varepsilon\left(\omega_{\mathbf{f}}, \mathbf{f}\right)\right|^{2}} \sum_{\mathbf{k}}\left\langle a_{\mathbf{k}+\frac{\mathrm{f}}{2}}^{+} a_{\mathbf{k}-\frac{\mathrm{f}}{2}} a_{\mathbf{k}-\frac{\mathrm{f}}{2}}^{+} a_{\mathbf{k}+\frac{\mathrm{f}}{2}}\right\rangle_{t} \delta\left(\Omega_{\mathbf{k}+\frac{\mathrm{f}}{2}}-\Omega_{\mathbf{k}-\frac{\mathrm{f}}{2}}-\omega_{\mathbf{f}}\right)\left(N_{\mathbf{f}}+1\right)- \\
& -\frac{2\left|C_{f}\right|^{2}}{V_{0} \hbar^{2}\left|\varepsilon\left(\omega_{\mathbf{f}}, \mathbf{f}\right)\right|^{2}} \sum_{\mathbf{k}}\left\langle a_{\mathbf{k}-\frac{\mathrm{f}}{2}}^{+} a_{\mathbf{k}+\frac{\mathrm{f}}{2}} a_{\mathbf{k}+\frac{\mathrm{f}}{2}}^{+} a_{\mathbf{k}-\frac{\mathrm{f}}{2}}\right\rangle_{t} \delta\left(\Omega_{\mathbf{k}+\frac{\mathrm{f}}{2}}-\Omega_{\mathbf{k}-\frac{\mathrm{f}}{2}}-\omega_{\mathbf{f}}\right) N_{\mathbf{f}}
\end{aligned}
$$


Taking into account the commutation rules for $a_{\mathbf{k}}^{+} a_{\mathbf{k}_{1}}$ it is easy to obtain

$$
\left\langle a_{\mathbf{k}}^{+} a_{\mathbf{k}_{1}} a_{\mathbf{k}_{1}}^{+} a_{\mathbf{k}}\right\rangle_{t}=F_{\mathbf{k}}(t)-\left\langle a_{\mathbf{k}}^{+} a_{\mathbf{k}_{1}}^{+} a_{\mathbf{k}_{1}} a_{\mathbf{k}}\right\rangle_{t} .
$$

According to the results from [1]

$$
\left\langle a_{\mathbf{k}}^{+} a_{\mathbf{k}_{1}}^{+} a_{\mathbf{k}_{1}} a_{\mathbf{k}}\right\rangle=F_{\mathbf{k}} F_{\mathbf{k}_{1}}+\phi\left(\mathbf{k}, \mathbf{k}_{1}\right)
$$

where $\phi\left(\mathbf{k}, \mathbf{k}_{1}\right)$ is a non-equilibrium correction to the pair correlation function.

Finally, the kinetic equation for phonons with non-equilibrium corrections of electron pair correlators takes the form:

$$
\begin{aligned}
\frac{d N_{\mathbf{f}}}{d t} & =\frac{2\left|C_{f}\right|^{2}}{V_{0} \hbar^{2}\left|\varepsilon\left(\omega_{f}, \mathbf{f}\right)\right|^{2}} \\
& \times \sum_{\mathbf{k}}\left[F_{\mathbf{k}+\frac{\mathbf{f}}{2}}\left(1-F_{\mathbf{k}-\frac{\mathbf{f}}{2}}\right)-\phi\left(\mathbf{k}+\frac{\mathbf{f}}{2}, \mathbf{k}-\frac{\mathbf{f}}{2}\right)\right]\left(N_{\mathbf{f}}+1\right) \delta\left(\Omega_{\mathbf{k}+\frac{\mathbf{f}}{2}}-\Omega_{\mathbf{k}-\frac{\mathbf{f}}{2}}-\omega_{\mathbf{f}}\right)- \\
& -\left[F_{\mathbf{k}-\frac{\mathbf{f}}{2}}\left(1-F_{\mathbf{k}+\frac{\mathbf{f}}{2}}\right)-\phi(\mathbf{k}-\mathbf{f} / 2, \mathbf{k}+\mathbf{f} / 2)\right] N_{\mathbf{f}} \delta\left(\Omega_{\mathbf{k}+\frac{\mathbf{f}}{2}}-\Omega_{\mathbf{k}-\frac{\mathbf{f}}{2}}-\omega_{\mathbf{f}}\right)
\end{aligned}
$$

Certainly, it needs to keep in mind the Eq. (4) describes the part of full time dependence of the phonon occupation numbers that resulting from electron-phonon interaction only.

It should be stressed that for ordinary classical equilibrium electron gas there are no pair correlations between different states of momentum space. This fact can be easily seen from the form of the Gibbs distribution. In conclusion of these considerations it needs to underline that the contribution of non-equilibrium correlations to kinetics should be expected in the cases when the particles considered are scattered by nonequilibrium many-particle system.

\section{ELECTRON ENERGY LOSS RATE VIA PHONONS}

The energy loss rate of electrons due to phonons per unit volume can be obtained using the relation

$$
P=\frac{1}{V_{0}} \sum_{\mathbf{f}} \hbar \omega_{\mathbf{f}} \frac{d N_{\mathbf{f}}}{d t} .
$$

It is seen from the kinetic equation for phonons Eq.(4) that the loss rate can be broken up into two parts:

$$
P=P_{1}+P_{2},
$$

where the first term on the right hand side is related to the one-particle electron distribution function and it is described by the known expressions. The second one is due to the correlation contribution determined by the function $\phi$.We assume during the calculations of $P$ the electron-electron collisions are sufficiently frequent in order to provide 
the use of the electron temperature approximation for hot electrons. As a result of these assumptions the following formulae may be derived

$$
\begin{aligned}
P_{1}\left(T, T_{0}\right) & =\sum_{\mathbf{f}} \frac{2\left|C_{f}\right|^{2}}{\left(V_{0} \hbar\right)^{2}\left|\varepsilon\left(\omega_{f}, \mathbf{f}\right)\right|^{2}} \hbar \omega_{f}\left[N_{\mathbf{f}}^{0}(T)-N_{\mathbf{f}}\right] \\
& \times \sum_{\mathbf{k}}\left[F_{\mathbf{k}}^{0}(T)-F_{\mathbf{k}+\mathbf{f}}^{0}(T)\right] \delta\left(\Omega_{\mathbf{k}+\mathbf{f}}-\Omega_{\mathbf{k}}-\omega_{\mathbf{f}}\right)
\end{aligned}
$$

and

$$
P_{2}\left(T, T_{0}\right)=-\sum_{\mathbf{f}} \frac{2\left|C_{f}\right|^{2}}{\left(V_{0} \hbar\right)^{2} \mid \varepsilon\left(\omega_{\mathbf{f}},\left.\mathbf{f}\right|^{2}\right.} \hbar \omega_{\mathbf{f}} \sum_{\mathbf{k}} \phi(\mathbf{k}+\mathbf{f}, \mathbf{k}) \delta\left(\Omega_{\mathbf{k}+\mathbf{f}}-\Omega_{\mathbf{k}}-\omega_{\mathbf{f}}\right)
$$

Here $N_{\mathbf{f}}^{0}(T)=\left[\exp \left(\hbar \omega_{\mathbf{f}} / T\right)-1\right]^{-1}$ is the equilibrium Bose-Einstein distribution with electron temperature $T$ and $N_{\mathrm{f}}$ is the true non-equilibrium phonon distribution function. Further, we neglect possible phonon heating and consider that the phonons remain in thermal equilibrium at temperature $T_{0}$, so $N_{\mathbf{f}}=N_{\mathbf{f}}^{0}\left(T_{0}\right)$.

The function $\phi\left(\mathbf{k}, \mathbf{k}_{1}\right)$ was found in [2] under accepted assumptions for the case of nondegenerate electron gas and can be presented in the form:

$$
\phi\left(\mathbf{k}, \mathbf{k}_{1}\right)=Q\left(T, T_{0}\right) \frac{\partial F_{\mathbf{k}}^{0}}{\partial T} \frac{\partial F_{\mathbf{k}_{1}}^{0}}{\partial T}
$$

where

$$
Q\left(T, T_{0}\right)=\frac{T^{2} N_{c}(T)}{n c_{e}}\left[\frac{P\left(T, T_{0}\right) /\left(T-T_{0}\right)}{d P / d T}-1\right] .
$$

Here $N_{c}(T)$ and $n c_{e}=3 n / 2$ are the effective density of states and specific heat capacitance of electron gas with concentration $n$, respectively. The expression for $\phi$ in [2] was obtained without accounting for the contribution of $\phi$ itself in $P$.Therefore, the quantity $P_{1}$ was only implied as the total energy loss rate. Now evidently we have the differential equation for determination of $P_{2}\left(T, T_{0}\right)$ or full $P$ since this quantity is defined by the right hand side of the equality Eq. (6) including $P_{2}\left(T, T_{0}\right)$ itself. The situation is quite resembling the self-consistent field approach in plasma theory. It needs to stress that the substitution of the total loss rate $P$ instead of $P_{1}$ in the expression for $\phi\left(k, k_{1}\right)$ is justified because all symmetry properties of the electron-electron and electron-phonon integrals of collisions used in the derivation are not destroyed by introducing $\phi\left(\mathbf{k}, \mathbf{k}_{1}\right)$ in collision integrals.

It is of interest to estimate the interrelation between $P_{1}$ and $P_{2}$. Instead carrying out the integrations in Eqs. (5)-(6) and following solution of a nonlinear differential equation for $P$ (or $P_{2}$ ) we can use the experimental data from Ref. [6] where hot-electron energy relaxation time at helium temperatures in $n-I n S b$ was investigated and all necessary information on $n, P, d P / d T$, and $T$ can be extracted from. It was shown there that at low heating the electron energy loss is due to acoustic phonon scattering and next it is changed by longitudinal optical (LO) phonon scattering. So we can evaluate $P_{2} / P_{1}$ for both types of the electron-phonon scattering. 
It is necessary to carry out preliminary the integrations over $\mathbf{k}$ in Eqs. (5)-(6) for all essential parameters be explicitly introduced. Taking into account nondegeneracy of electron gas the expressions for $P_{1}$ and $P_{2}$ can be transform as following

$$
\begin{aligned}
P_{1}\left(T, T_{0}\right) & =\left(T-T_{0}\right) \frac{2 m s^{2}}{(2 \pi)^{2} \hbar^{3}} \sum_{\mathbf{f}} \frac{2\left|C_{f}\right|^{2}}{\hbar V_{0}\left|\varepsilon\left(\omega_{f}, \mathbf{f}\right)\right|^{2}} F_{\mathbf{f} / 2}^{0}(T) \\
P_{2}\left(T, T_{0}\right) & = \pm \frac{9 T m^{2} s}{4 c_{e} \hbar^{3}}\left[\frac{P\left(T, T_{0}\right) /\left(T-T_{0}\right)}{d P / d T}-1\right] \sum_{\mathbf{f}} \frac{2\left|C_{f}\right|^{2}}{\hbar V_{0}\left|\varepsilon\left(\omega_{f}, \mathbf{f}\right)\right|^{2}} F_{\mathbf{f} / 2}^{0}(T / 2)[1+O(T)] \\
F_{\mathbf{k}}^{0}(T) & =\frac{n}{N_{c}(T)} \exp \left(-\frac{\hbar^{2} k^{2}}{2 m T}\right) .
\end{aligned}
$$

for acoustic phonon scattering. Here $s$ is the sound velocity and it has been accounted for the near-elastic scattering of electrons by acoustic phonons. While the sign of expression (9) for $P_{2}$ leaves indeterminate since implicit $O(T)$ terms may redefine it, the order of magnitude of $P_{2} / P_{1}$ can be found. The expression Eq.(8) is just the Kogan's formula from [5] corrected for the screening of electron-phonon interaction.

In the case of LO phonon scattering we obtain

$$
\begin{aligned}
P_{1}\left(T, T_{0}\right) & =\frac{m^{2} T \hbar \omega_{L O}}{\pi^{2} \hbar^{4}} \frac{n}{N_{c}(T)}\left(1-\mathrm{e}^{-\hbar \omega_{L O} / T}\right)\left[N_{\mathbf{f}}^{0}(T)-N_{\mathbf{f}}^{0}\left(T_{0}\right)\right] \\
& \times \sum_{\mathbf{f}} \frac{2\left|C_{f}\right|^{2}}{\hbar V_{0}\left|\varepsilon\left(\omega_{f}, \mathbf{f}\right)\right|^{2} f} \exp \left(-\frac{\hbar^{2}}{2 m T}\left[\frac{f^{2}-f_{L O}^{2}}{2 f}\right]^{2}\right) \\
P_{2}\left(T, T_{0}\right)= & \pm \frac{m^{2} \hbar^{2} \omega_{L O}^{2}}{2(2 \pi)^{2} \hbar^{4}} \frac{n}{N_{c}(T)}\left[\frac{P\left(T, T_{0}\right) /\left(T-T_{0}\right)}{d P / d T}-1\right] \exp \left(-\frac{\hbar \omega_{L O}}{T}\right) \\
& \times \sum_{\mathbf{f}} \frac{2\left|C_{f}\right|^{2}}{\hbar V_{0}\left|\varepsilon\left(\omega_{f}, \mathbf{f}\right)\right|^{2} f} \exp \left(-\frac{2 \hbar^{2}}{2 m T}\left[\frac{f^{2}-f_{L O}^{2}}{2 f}\right]^{2}\right)[1+O(T)]
\end{aligned}
$$

where $f_{L O}^{2}=2 m \omega_{L O} / \hbar$.

The sums over $\mathbf{f}$ in corresponding expressions are of the same order of magnitude. Therefore, we have to evaluate following ratios

$$
\frac{P_{2}}{P_{1}}=\frac{3 \pi^{2} T}{T-T_{0}}\left[\frac{P\left(T, T_{0}\right) /\left(T-T_{0}\right)}{d P / d T}-1\right]
$$

for the acoustic phonon scattering and

$$
\frac{P_{2}}{P_{1}}=\frac{\hbar \omega_{L O}}{8 T}\left[\frac{P\left(T, T_{0}\right) /\left(T-T_{0}\right)}{d P / d T}-1\right]
$$

for the low-temperature LO phonon scattering $\left(\hbar \omega_{L O} / T>>1\right)$. Turning to experimental data [6] and using relation between $d P / d T$ and measured electron energy relaxation 
time $\tau_{P}=n c_{e} /(d P / d T)$ we obtain for the acoustic scattering $P_{2} / P_{1} \simeq 1.3$ at $T \simeq 7.8 K$, $P \simeq 6 \cdot 10^{-3} \mathrm{~W} / \mathrm{cm}^{3}, \tau_{P} \simeq 4 \cdot 10^{-7} \mathrm{~s}$ and $P_{2} / P_{1} \simeq 0.6$ at $T \simeq 15.8 \mathrm{~K}, P \simeq 3.8 \cdot 10^{-2} \mathrm{~W} / \mathrm{cm}^{3}$, $\tau_{P} \simeq 0.8 \cdot 10^{-7} s$ for LO phonon scattering. All data are taken at $T_{0}=4.2 \mathrm{~K}$. These estimations may be considered as an indication that the pair correlations contribute to hot electron energy loss via phonons comparable with common accepted expressions.

\section{Concluding remarks}

It is of importance to note in conclusion that the expression for $\phi\left(k, k_{1}\right)$ found in the limit of high frequency of the electron-electron collisions does contain coupling constants of neither electron-electron nor electron-phonon interactions. Therefore, the corresponding corrections to the integral of phonon-electron collisions do not contain additional powers of the small parameters of the kinetic theory. The interesting but still open question: are there analogous contributions to the hydrodynamic equations?

\section{ACKNOWLEDGMENTS}

This work was partially supported by Russian Foundation for Basic Researches.

\section{REFERENCES}

1. S.V. Gantsevich, V.L. Gurevich, and R. Katilius. FTT 11, 308 (1969) [Sov. Phys. Sol. State 11, 247 (1969)]

2. A.Ya. Shul'man. FTT 12, 1181 (1970) [Sov. Phys. Sol. State 12, 922 (1970)]

3. A.Ya. Shul'man. cond-mat/0212595

4. A.Ya. Shul'man. In: Progress in Nonequilibrium Green's Functions-2, eds. M. Bonitz and D. Semkat. World Scientific Publishing, Singapore, p. 74 (2003)

5. Sh.M. Kogan. FTT 4, 2474 (1962) [Sov. Phys. Sol. State 4, 1813 (1963)]

6. T.M. Lifshits, A.Ya. Oleinikov, and A.Ya. Shul'man. Phys. Stat. Sol. 14, 511 (1966) 